\title{
LAS EMOCIONES Y SU RELACIÓN CON LAS INTELIGENCIAS MÚLTIPLES EN LAS ASIGNATURAS DE CIENCIAS Y MATEMÁTICAS EN SECUNDARIA
}

\author{
Mercedes Gómez Acuñas \\ mergomez@unex.es \\ Cristina Lucas Millán \\ María Luisa Bermejo García \\ mbermejo@unex.es \\ María José Rabazo Méndez \\ Facultad de Educación. Universidad de Extremadura.
}

Fecha de Recepción: 23 Febrero 2018

Fecha de Admisión: 10 Abril 2018

\section{RESUMEN}

En la actualidad los aspectos racionales y emocionales se interrelacionan entre sí, ya que las emociones influyen en los pensamientos al igual que los procesos cognitivos influyen en los estados emocionales (Mayer, Salovey, Caruso y Cherkasskiy, 2011).

En este estudio hemos analizado las emociones del alumnado en las asignaturas de Ciencias impartidas en tercero de la ESO. La muestra ha sido de 46 chicas y 34 chicos, pertenecientes a dos IES de la provincia de Badajoz. Para su análisis se ha elaborado un cuestionario para conocer las emociones que presentan en Física y Química, Matemáticas y Ciencias Naturales, relacionándolas con la teoría de las inteligencias múltiples (Gardner, 1998), ya que el alumnado trabaja y aprende en función de sus inteligencias. La metodología utilizada en esta investigación ha sido cuantitativa.

Entre los resultados obtenidos están los siguientes: Los alumnos de tercero de ESO presentan pocas emociones positivas en la asignatura de Física y química, y, muestran mayores puntuaciones en emociones negativas como preocupación y nerviosismo. En la asignatura de matemáticas presentan un alto porcentaje en emociones positivas (confianza y tranquilidad), y también de emociones negativas (preocupación). Ciencias Naturales es la asignatura que más le gusta de las tres y manifiestan emociones positivas como alegría, confianza, tranquilidad y satisfacción.

Existe una correlación positiva en las puntuaciones más altas en emociones positivas en Ciencias Naturales y una mayor puntuación en inteligencia Interpersonal y Naturalista.

Existen correlaciones significativas entre las inteligencias múltiples Lógico Matemática y Espacial y las variables relacionadas con las emociones positivas en Física y Química, en Matemáticas y en Ciencias Naturales.

Palabras claves: emociones; inteligencias múltiples; asignaturas de ciencias; secundaria 


\title{
LAS EMOCIONES Y SU RELACIÓN CON LAS INTELIGENCIAS MÚLTIPLES EN LAS ASIGNATURAS DE CIENCIAS Y MATEMÁTICAS EN SECUNDARIA
}

\author{
ABSTRACT \\ Emotions and their relationship with multiple intelligences in the subjects of science and \\ mathematics in Secondary.
}

At present the rational and emotional aspects are interrelated, since emotions influence thoughts just as cognitive processes influence emotional states (Mayer, Salovey, Caruso and Cherkasskiy, 2011).

In this study we analyzed the emotions of students in the subjects of Sciences taught in third of the ESO. The sample was of 46 girls and 34 boys, belonging to two IES of the province of Badajoz. For their analysis a questionnaire has been developed to know the emotions they present in Physics and Chemistry, Mathematics and Natural Sciences, relating them to the theory of multiple intelligences (Gardner, 1998), since students work and learn according to their intelligences. The methodology used in this research has been quantitative.

Among the results obtained are the following:

ESO students present few positive emotions in the subject of Physics and Chemistry, and show higher scores in negative emotions such as worry and nervousness. In the subject of mathematics they present a high percentage of positive emotions (confidence and tranquility), and also of negative emotions (worry). Natural Sciences is the subject that you like the most of the three and manifest positive emotions such as joy, confidence, tranquility and satisfaction.

There is a positive correlation in the highest scores in positive emotions in Natural Sciences and a higher score in Interpersonal and Naturalist Intelligence.

There are significant correlations between the multiple mathematical and spatial logical intelligences and the variables related to positive emotions in Physics and Chemistry, in Mathematics and in Natural Sciences.

Keywords: emotions; multiple intelligences; science subjects; high school

\section{INTRODUCCIÓN}

Hace unos años, las investigaciones sobre los conceptos de emoción y cognición se desarroIlaban de manera paralela, e incluso, se consideraban como opuestos. Predominaba el lado racional de la mente menoscabando el aspecto emocional y se creía que la inteligencia era necesaria para dominar las emociones y sentimientos. En la actualidad los aspectos racionales y emocionales se interrelacionan entre sí, y se considera que lo cognitivo configura lo afectivo y lo afectivo lo cognitivo (Dávila, Borrachero, Brígido, Costillo 2014). Cuando ambos se utilizan juntos, el proceso de aprendizaje se puede construir con unas bases más firmes.

Hemos intentado relacionar las emociones con las inteligencias múltiples de Gadner (1983). En esta teoría se afirma que todos los seres humanos poseen todas las inteligencias pero que cada una de ellas se manifiesta de manera diferente en cada persona. Según Gadner (1995) se establecen 8 tipos de inteligencias: musical, cinético-corporal, lógico-matemática, lingüística, espacial, interpersonal e intrapersonal; se añade posteriormente la inteligencia naturalista y existencial.

La inteligencia es una destreza que se puede desarrollar y en cada campo se demanda un aspecto distinto de la inteligencia. El surgimiento de la inteligencia emocional tiene como base al carácter multifactorial de las inteligencias, es decir las inteligencias múltiples (Gardner,2005).

En esta investigación hemos querido comprobar las emociones que el alumnado presenta en las asignaturas de Ciencias en tercero de la ESO y su relación con las inteligencias múltiples.

\section{Emoción}

Definir lo que es emoción es un proceso que presenta mucha dificultad. Para FernándezBerrocal y Ramos (2005), la emoción es el sistema menos conocido de los procesos cognitivos 
básicos. Pero en general hay bastante acuerdo en considerar que una emoción es un estado complejo del organismo caracterizado por una excitación o perturbación que se genera como respuesta a un acontecimiento externo o interno. Las emociones según Bisquerra 2000:61) se definen "como reacciones subjetivas asociadas a cambios fisiológicos y conductuales."

Las emociones sirven para mejorar el pensamiento porque se pone mayor atención a la información significativa de forma más rápida. Facilitan el cambio de perspectiva, la formación de juicios y la consideración de nuevos puntos de vista de los problemas debido a las continuas variaciones emocionales. Por ejemplo, ser capaz de anticipar cómo nos sentiríamos frente a un suceso, puede ayudar a elegir decisiones acertadas en el momento oportuno (Damasio,2006).

Numerosos autores han intentado realizar una clasificación de las emociones, sin embargo, Goleman (2000:419) afirma que "La verdad es que en este terreno no hay respuestas claras y el debate científico sobre la clasificación de las emociones aún se halla en el tapete".

La clasificación más utilizada consiste en distinguir entre las emociones básicas y emociones complejas o secundarias, positivas y negativas. Las emociones básicas son denominadas primarias, y se reconocen por su expresión facial. Ekman (1992) descubrió que estas emociones que no son aprendidas, se manifiestan en expresiones faciales reconocidas por las demás culturas, son universales. Podemos destacar como emociones básicas: alegría, tristeza, ira, miedo, sorpresa y asco. Estas emociones están presentes en todas las culturas.

Como exponer las distintas catalogaciones sería algo complejo, nos limitamos a nombrar en el siguiente cuadro las emociones que analizamos en nuestro estudio.

Tabla 1: Lista de emociones descritas en nuestro estudio

\begin{tabular}{|l|l|l|}
$\begin{array}{l}\text { Emociones } \\
\text { positivas }\end{array}$ & $\begin{array}{l}\text { Emociones } \\
\text { negativas }\end{array}$ & $\begin{array}{l}\text { Emociones } \\
\text { ambiguas }\end{array}$ \\
\hline Alegría & Preocupación & Sorpresa \\
\hline Confianza & Vergüenza & \\
\hline Felicidad & Miedo & \\
\hline Admiración & Tristeza & \\
\hline Tranquilidad & Ira & \\
\hline Satisfacción & Nerviosismo & \\
\hline Entusiasmo & & \\
\hline
\end{tabular}

\section{INTELIGENCIAS MÚLTIPLES Y LA INTELIGENCIA EMOCIONAL}

Gardner (1995), afirma que todo individuo tiene los ocho tipos de inteligencia mencionados, aunque una persona puede destacar más en una inteligencia que en otras. Según su opinión, es absurdo que se siga insistiendo en que todos los alumnos aprendan de la misma manera. La misma materia se podría presentar de formas muy diversas que permitieran al alumno asimilarla partiendo de sus capacidades y aprovechando sus puntos fuertes. Este enfoque permitiría estimular las capacidades de los alumnos según sus potencialidades y competencias.

Se consideran la inteligencia interpersonal (la capacidad para comprender las intenciones, motivaciones y deseos de otras personas) y la inteligencia intrapersonal (la capacidad para comprenderse a sí mismo, percibir y/o autorregular los sentimientos, temores y motivaciones) precursoras de la inteligencia emocional.

Greenspan (1998:15), sostiene: "Las emociones, y no la estimulación cognitiva, constituyen los cimientos de la arquitectura mental primaria". Este autor argumenta que el papel más decisivo de las emociones consiste en crear, organizar y coordinar muchas de las más importantes funciones cerebrales. 


\section{LAS EMOCIONES Y SU RELACIÓN CON LAS INTELIGENCIAS MÚLTIPLES EN LAS ASIGNATURAS DE CIENCIAS Y MATEMÁTICAS EN SECUNDARIA}

Salovey y Mayer (1990) exponen que a veces las emociones se presentan como interrupciones que desorganizan la actividad mental. Argumentan que esta visión es equivocada porque las situaciones emocionales intensas, en ocasiones, estimulan la inteligencia, ayudando a los individuos a priorizar los procesos del pensamiento. Por tanto, más que interrumpir el pensamiento lógico, las emociones ayudan a un mejor pensamiento. Goleman (1996) afirma que emoción e inteligencia actúan en armonía. Este autor preconiza la inteligencia emocional como un importante factor de éxito, y se basa esta inteligencia en la capacidad para conocer, controlar e inducir emociones y estados de ánimo, tanto en uno mismo como en los demás.

\section{OBJETIVOS DE LA INVESTIGACIÓN}

Los objetivos que nos hemos planteado en este estudio son los siguientes:

Conocer las emociones positivas o negativas, que presentan los alumnos, en las distintas asignaturas de Ciencia de tercero de la ESO, en Física y Química, Matemáticas y Ciencias Naturales.

Confirmar que no existe una única inteligencia, sino que el alumnado trabaja y aprende en función de sus inteligencias múltiples.

\section{METODOLOGÍA.}

La metodología utilizada en esta investigación ha sido cuantitativa, el instrumento utilizado es un cuestionario adaptado por nosotros. Para poder comprobar los resultados del cuestionario, hemos trabajado con el programa SPSS 18.

\section{MUESTRA}

Está compuesta la muestra por 80 sujetos perteneciente a dos Institutos Públicos que imparten la Enseñanza Secundaria Obligatoria y de Bachillerato; uno está ubicado en la ciudad de Badajoz y el otro en la provincia de Badajoz. Pertenecen 40 alumnos al IES de la provincia de Badajoz y otros 40 alumnos al IES de la capital. El total de los alumnos lo forman 46 chicas y 34 chicos.

\section{INSTRUMENTO}

Para llevar a cabo nuestro estudio se ha utilizado una parte del cuestionario (Borrachero, 2015) y ha sido adaptado para la realización de nuestra investigación.

A continuación haremos una breve descripción sobre el cuestionario:

En primer lugar, se describen los datos personales del alumnado, siempre teniendo presente que se trata de un cuestionario anónimo (Lucas 2013). Otras preguntas que se plantean son las notas que han obtenido el alumnado en $3^{\circ}$ de la ESO en las asignaturas de ciencias y matemáticas.

En segundo lugar, hemos creído conveniente seleccionar algunas emociones que puede presentar el alumnado con respecto a las asignaturas de Ciencias Naturales, Física y Química y Matemáticas.

Y en tercer lugar, les hemos planteado preguntas para conocer las inteligencias múltiples.

\section{ANÁLISIS DE DATOS}

\section{Análisis descriptivo de las variables sociodemográficas}

En este apartado, presentaremos los análisis descriptivos del cuestionario. Entre otras preguntas hemos planteado la edad, el género y la nota que han obtenido en las asignaturas de Ciencias en tercero de la ESO en el primer trimestre.

\section{Género}

El cuestionario se le ha pasado a un total de 80 sujetos, siendo un 42,5\% (34 personas) chicos y un $57,5 \%$ (46 personas) chicas. 
Tabla2: Porcentaje de la muestra en relación al género.

\begin{tabular}{|ll|l|l|l|l|}
\hline & & Frecuencia & Porcentaje & $\begin{array}{l}\text { Porcentaje } \\
\text { válido }\end{array}$ & $\begin{array}{l}\text { Porcentaje } \\
\text { acumulado }\end{array}$ \\
\hline Válidos & Chico & 34 & 42,5 & 42,5 & 42,5 \\
& Chica & 46 & 57,5 & 57,5 & 100,0 \\
& Total & 80 & 100,0 & 100,0 & \\
\hline
\end{tabular}

Las edades de los alumnos están comprendidas entre los 14 a más de 16 años, siendo más frecuente los que tienen entre $15-16$ años $(68,8 \%)$.

Tabla 3: Porcentaje de la muestra en relación a la edad.

\begin{tabular}{|ll|l|l|l|l|}
\hline & & Frecuencia & Porcentaje & $\begin{array}{l}\text { Porcentaje } \\
\text { válido }\end{array}$ & $\begin{array}{l}\text { Porcentaje } \\
\text { acumulado }\end{array}$ \\
\hline Válidos & Menos de 14 años & 1 & 1,3 & 1,3 & 1,3 \\
& $14-15$ años & 19 & 23,8 & 23,8 & 25,0 \\
& $15-16$ años & 55 & 68,8 & 68,8 & 93,8 \\
& Mayor de 16 años & 5 & 6,3 & 6,3 & 100,0 \\
& Total & 80 & 100,0 & 100,0 & \\
\hline
\end{tabular}

Nota media obtenida en las asignaturas de ciencias y matemáticas Nota media en ciencias naturales

Tabla 4: Porcentaje de la muestra en relación a la nota media en este trimestre en Ciencia Naturales.

\begin{tabular}{|ll|l|l|l|l|}
\hline & & Frecuencia & Porcentaje & $\begin{array}{l}\text { Porcentaje } \\
\text { válido }\end{array}$ & $\begin{array}{l}\text { Porcentaje } \\
\text { acumulado }\end{array}$ \\
\hline Válidos & Menos de 5 & 9 & 11,3 & 11,3 & 11,3 \\
& Entre 5 a 6 & 34 & 42,5 & 42,5 & 53,8 \\
& Entre 7 a 8 & 19 & 23,8 & 23,8 & 77,5 \\
& Más de 8 & 17 & 21,3 & 21,3 & 98,8 \\
10 & 1 & 1,3 & 1,3 & 100,0 \\
Total & 80 & 100,0 & 100,0 & \\
& & & & \\
\hline
\end{tabular}

La nota media que han obtenido en este trimestre en la asignatura de Ciencias Naturales destacaremos con un 42,5\% (34 estudiantes) entre 5 a 6, 23,8\% (19 estudiantes ) entre 7 a 8, 21,2\% (17) y más de 8 y un $11,2 \%$ han sacado menos de 5 .

Nota media en Física y Química

En la pregunta de cuál ha sido la nota media que han sacado en este trimestre en la asignatura de Física y Química es de 40\% (32 estudiantes) un suspenso, 27,5\% (22) entre 5 a 6 y 16,2\% (13 estudiantes) entre 7 a 8 y más de 8.

Tabla 5: Porcentaje de la muestra en relación a la nota media en este trimestre en Física y Química.

\begin{tabular}{|ll|l|l|l|l|}
\hline & & & $\begin{array}{l}\text { Porcentaje } \\
\text { válido }\end{array}$ & $\begin{array}{l}\text { Porcentaje } \\
\text { acumulado }\end{array}$ \\
\hline Válidos & Menos de 5 & 32 & 40,0 & 40,0 & 40,0 \\
& Entre 5 a 6 & 22 & 27,5 & 27,5 & 67,5 \\
Entre 7 a 8 & 13 & 16,3 & 16,3 & 83,8 \\
Más de 8 & 13 & 16,3 & 16,3 & 100,0 \\
Total & 80 & 100,0 & 100,0 & \\
\hline
\end{tabular}


Nota media en Matemáticas

Cuándo le preguntamos por la nota media que han sacado en este trimestre en la asignatura de Matemáticas los resultados fueron los siguientes: $32,2 \%$ (26 sujetos) obtuvieron menos de 5 , $27,5 \%$ (22 alumnos) entre 5 a 6 , y un 23,8\% (19 s) entre 7 a 8.

Tabla 6: Porcentaje de la muestra en relación a la nota media en este trimestre en Matemáticas.

\begin{tabular}{|ll|l|l|l|l|}
\hline & & Frecuencia & Porcentaje & $\begin{array}{l}\text { Porcentaje } \\
\text { válido }\end{array}$ & $\begin{array}{l}\text { Porcentaje } \\
\text { acumulado }\end{array}$ \\
\hline Válidos & Menos de 5 & 26 & 32,5 & 32,5 & 32,5 \\
& Entre 5 a 6 & 22 & 27,5 & 27,5 & 60,0 \\
& Entre 7 a 8 & 19 & 23,8 & 23,8 & 83,8 \\
& Más de 8 & 9 & 11,3 & 11,3 & 95,0 \\
10 & 4 & 5,0 & 5,0 & 100,0 \\
Total & 80 & 100,0 & 100,0 & \\
\hline
\end{tabular}

\section{Analisis de datos}

\section{Analisis descriptivo de emociones y de inteligencias múltiples}

En este apartado describimos los resultados que han obtenido los alumnos de tercero de la ESO Análisis descriptivo de las emociones en las asignaturas de:

Física y Química.

Emociones Positivas:

Figura 1: Representación gráfica de la muestra según las emociones positivas en Física y Química.

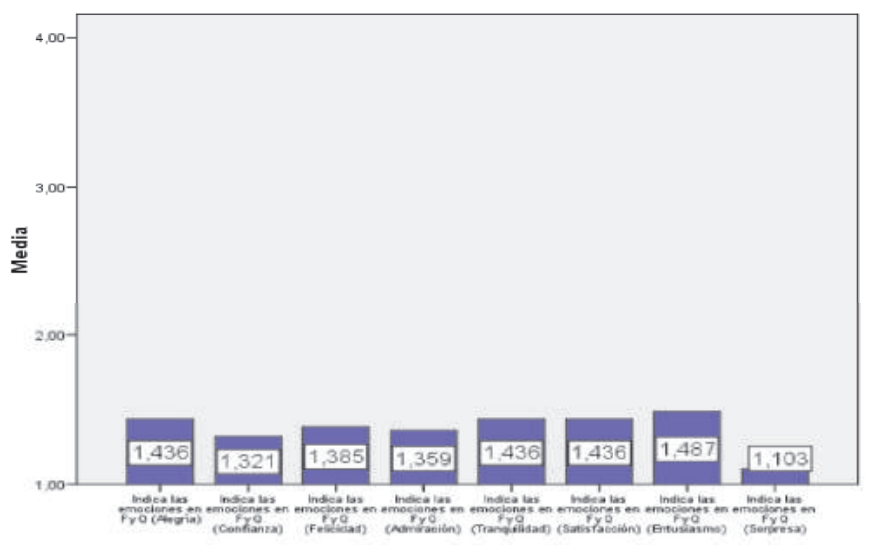

El alumnado no suele presentar emociones positivas en la asignatura de Física y Química, por lo que significa que no les gusta demasiado esta asignatura. La emoción con mayor puntuación es en Entusiasmo, con un 1,487\%, siguiéndoles muy de cerca con 1,436\% Alegría, Tranquilidad y Satisfacción.

Emociones negativas:

Al contrario del apartado anterior, sí comprobamos que presentan emociones negativas con res- 
pecto a la asignatura de Física y Química y sobresale la emoción de Preocupación con 1,738\%, la segunda más destacada Nerviosismo con 1,488\% y la tercera Vergüenza con un 1,088\%.

Figura 2. Representación gráfica de la muestra según las emociones negativas en Física y Química.

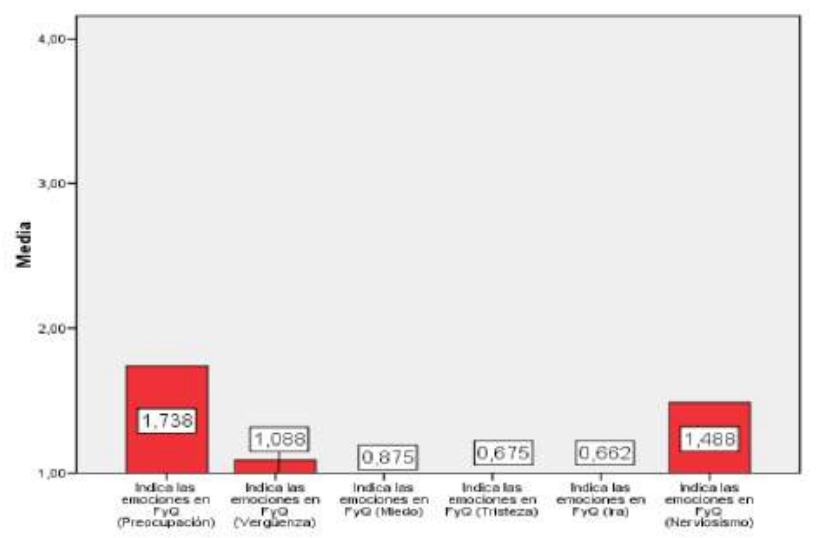

Por lo general los alumnos presentan un mayor número de emociones negativas que positivas con respecto a la asignatura de Física y química.

Matemáticas:

Emociones Positivas:

El alumnado de tercero de la ESO no suele presentar emociones positivas en la asignatura de Matemáticas, por lo que nos da a conocer que no les gusta esta asignatura, aunque son porcentajes más positivos que los que se presentan en Física y Química. Presentan un porcentaje de 1,641\% en Confianza y Tranquilidad 1,538\%. Las demás están alrededor en 1,4\%, menos Sorpresa que $1,01 \%$.

Figura 3: Representación gráfica de la muestra según las emociones positivas en matemáticas

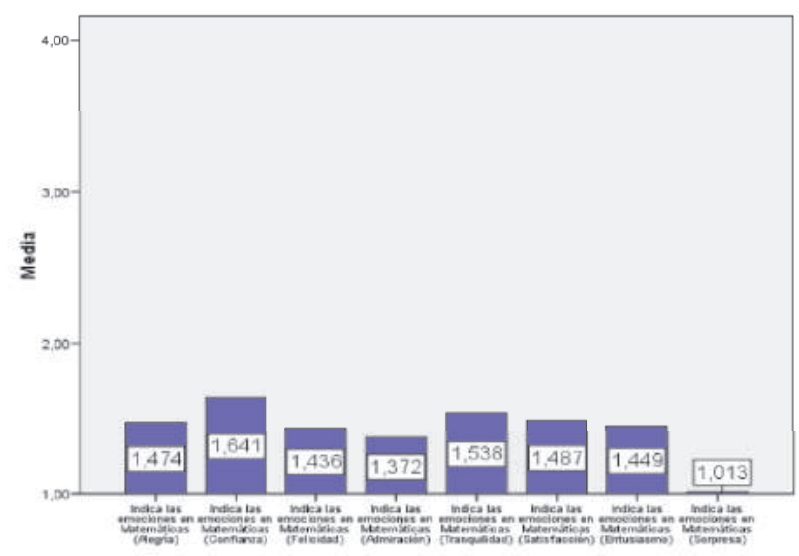


Matemáticas

Emociones Negativas

Comprobamos que presentan emociones negativas con respecto a la asignatura de Matemáticas. Las más destacadas son Preocupación con un 1,456\% y Nerviosismo con 1,304\%. Las demás, no sobre pasan el 1\%.

Figura 4: Representación gráfica de la muestra según las emociones negativas en matemáticas

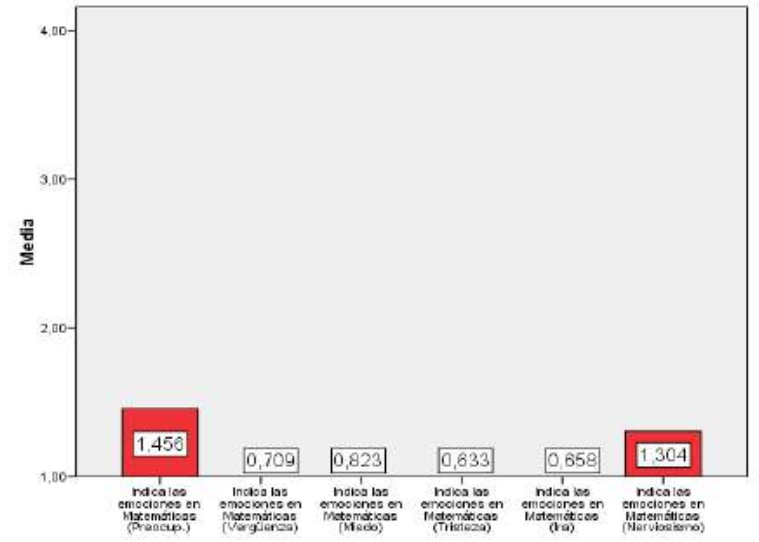

En definitiva, como se expone que el alumnado presenta más emociones negativas que positivas con respecto a la asignatura de Matemáticas.

En Ciencias Naturales:

Emociones Positivas:

El alumnado presenta emociones positivas en la asignatura de Ciencias Naturales, por lo que podemos comprobar que es la asignatura que más le gusta de las tres encuestadas. Todas las emociones rondan el 1,9\% 0 1,8\%, excepto admiración que presenta un 1,597\% y Sorpresa un 1,95\%.

Figura 5: Representación gráfica de la muestra según las emociones positivas en Ciencias Naturales.

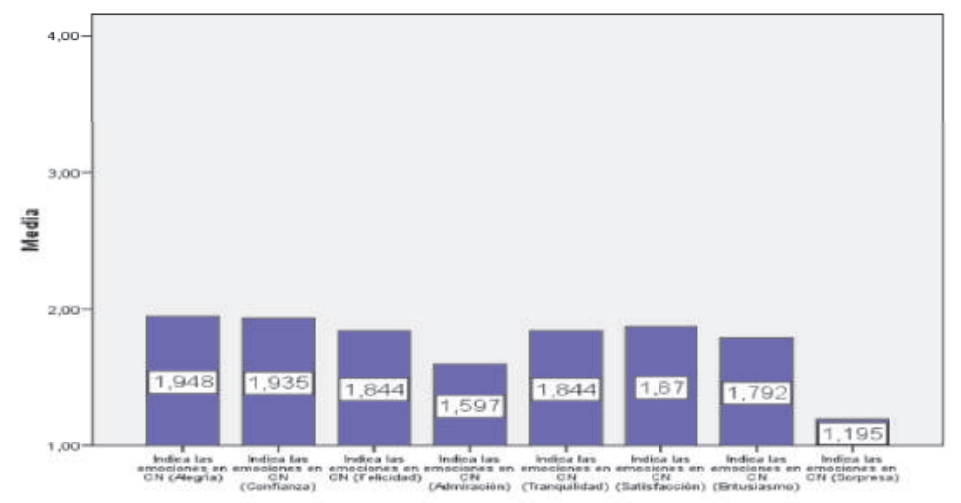


Emociones Negativas:

Estos alumnos suelen mostrar emociones negativas en relación a la asignatura de Ciencias Naturales. Tan solo Preocupación con un 1, 165\% y Nerviosismo un 1,101\%. Las demás emociones no superan el $1 \%$.

Figura 6: Representación gráfica de la muestra según las emociones negativas en Ciencias Naturales.

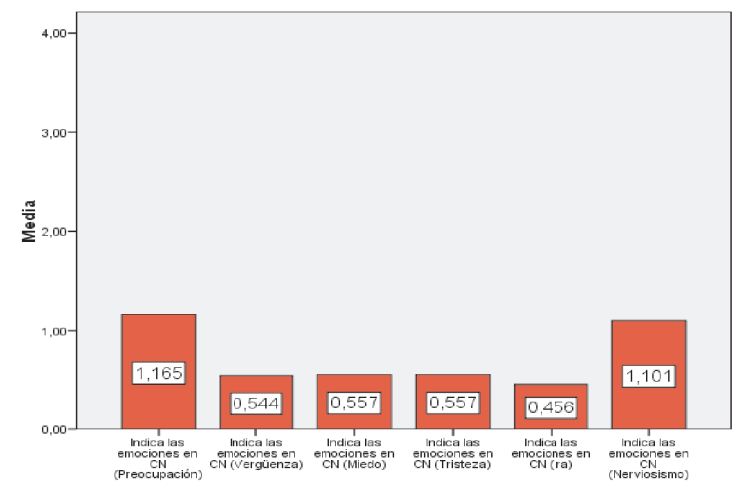

En definitiva, se pone de manifiesto que el alumnado presenta más emociones positivas que negativas con respecto a la asignatura de Ciencias Naturales.

\section{Análisis descriptivo de los tipos de inteligencias múltiples}

Seguidamente, vamos a realizar el análisis descriptivo de los resultados alcanzados por los alumnos en los tipos de inteligencias múltiples

Figura 7: Representación gráfica de la muestra según las inteligencias múltiples.

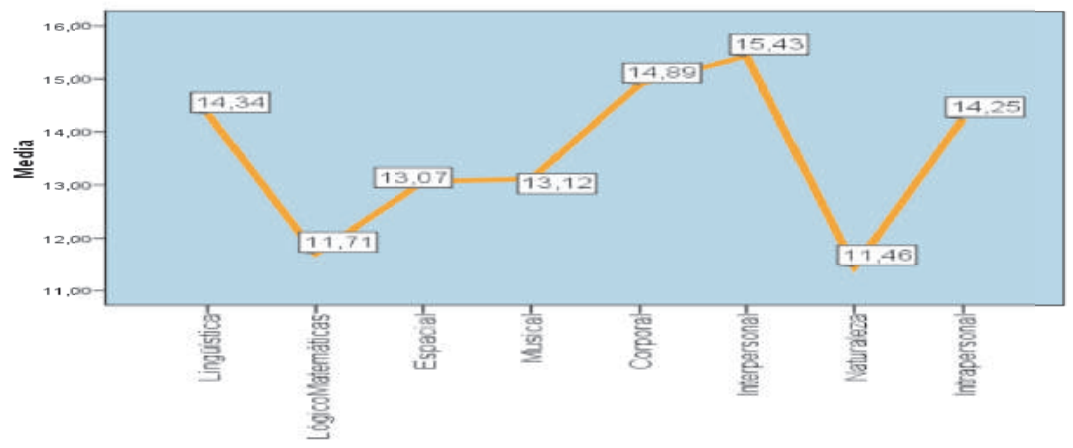

En la figura 7 se exponen los resultados de las 8 inteligencias múltiples obtenidos en los cuestionarios realizados por los alumnos. Podemos observar como la inteligencia interpersonal ha sido la que mayor puntuación ha obtenido, siguiéndoles de cerca la inteligencia corporal, lingüística e intrapersonal. 


\section{Análisis inferencial.}

En este estudio hemos incluido una hipótesis sobre la relación del tipo de inteligencias múltiples en relación a las emociones de los alumnos. La hipótesis es la siguiente

Las mayores puntuaciones en algunos tipos de inteligencias múltiples están asociadas a experimentar emociones positivas en las asignaturas de Física y Química, Matemáticas y Ciencias Naturales.

Hacemos un análisis estadístico descriptivo:

Tabla7: Porcentaje de la muestra en relación a las inteligencias múltiples asociadas a experimentar emociones positivas en Física y Química, Matemáticas y Ciencias Naturales.

\begin{tabular}{|l|l|l|l|}
\hline & Media & $\begin{array}{l}\text { Desviación } \\
\text { típica }\end{array}$ & $\mathrm{N}$ \\
\hline Lingüística & 14,3671 & 3,10573 & 79 \\
LógicoMatemática & 11,6835 & 3,23659 & 79 \\
S & 13,1000 & 2,77238 & 80 \\
Espacial & 13,0125 & 3,30513 & 80 \\
Musical & 14,8861 & 2,76882 & 79 \\
Corporal & 15,5125 & 2,49553 & 80 \\
Interpersonal & 11,5823 & 3,01112 & 79 \\
Naturaleza & 14,2250 & 2,54068 & 80 \\
Intrapersonal & 10,9615 & 4,30476 & 78 \\
EmocionPFQ & 11,4103 & 4,70544 & 78 \\
EmocionesPM & 14,0260 & 4,67348 & 77 \\
EmocionesPCN & \multicolumn{3}{|l}{} \\
\hline
\end{tabular}

Para que podamos lograr la hipótesis planteada hemos realizado un análisis de "Correlación de Pearson" con la finalidad de saber si las variables de inteligencias múltiples y las variables de las emociones positivas (en Física y Química, Matemáticas y Ciencias Naturales) están relacionadas entre sí: 
Tabla 8: Correlación de la muestra en relación a las inteligencias múltiples asociadas a experimentar emociones positivas en Física y Química, Matemáticas y Ciencias Naturales.

\begin{tabular}{|c|c|c|c|c|}
\hline & & EmocionesP FQ & EmocionesPM & EmocionesPCN \\
\hline \multirow[t]{3}{*}{ Lingüística } & Correlación de Pearson & 183 &, 126 & 221 \\
\hline & Sig. (bilateral) &, 111 & 277 & 055 \\
\hline & $\mathrm{N}$ & 77 & 77 & 76 \\
\hline \multirow[t]{3}{*}{ Lógico Matemáticas } & Correlación de Pearson &, $489(* *)$ &, $596(* *)$ & ,260(*) \\
\hline & Sig. (bilateral) &, 000 &, 000 &, 023 \\
\hline & $\mathrm{N}$ & 77 & 77 & 76 \\
\hline \multirow[t]{3}{*}{ Espacial } & Correlación de Pearson &, $365(* *)$ &, $350(* *)$ &, $333(* *)$ \\
\hline & Sig. (bilateral) &, 001 &, 002 &, 003 \\
\hline & $\mathrm{N}$ & 78 & 78 & 77 \\
\hline \multirow[t]{3}{*}{ Musical } & Correlación de Pearson &,- 108 &,- 102 & ,212 \\
\hline & Sig. (bilateral) & ,348 & ,375 & 064 \\
\hline & $\mathrm{N}$ & 78 & 78 & 77 \\
\hline \multirow[t]{3}{*}{ Corporal } & Correlación de Pearson & ,089 &, 121 &, $363(* *)$ \\
\hline & Sig. (bilateral) & ,443 & ,296 &, 001 \\
\hline & $\mathrm{N}$ & 77 & 77 & 77 \\
\hline \multirow[t]{3}{*}{ Interpersonal } & Correlación de Pearson &, 143 &, 036 &, 032 \\
\hline & Sig. (bilateral) & ,213 &, 753 & ,785 \\
\hline & $\mathrm{N}$ & 78 & 78 & 77 \\
\hline \multirow[t]{3}{*}{ Naturaleza } & Correlación de Pearson &, 122 &, $260(*)$ &, $477(* *)$ \\
\hline & Sig. (bilateral) & ,285 & ,022 &, 000 \\
\hline & $\mathrm{N}$ & 78 & 77 & 76 \\
\hline \multirow[t]{3}{*}{ Intrapersonal } & Correlación de Pearson & ,216 &, 113 &, $229(*)$ \\
\hline & Sig. (bilateral) &, 058 & ,324 & 046 \\
\hline & $\mathrm{N}$ & 78 & 78 & 77 \\
\hline
\end{tabular}

En el resultado de la correlación que observamos en la tabla 4: existen correlaciones significativas entre las inteligencias múltiples Lógico Matemáticas y las variables emociones positivas en Física y Química $r=0,489(p=0,000)$, emociones positivas en Matemáticas $r=0,596(P=0,000)$ y emociones positivas en Ciencias Naturales $r=0,260(p=0,023)$.

También podemos observar correlaciones significativas entre las inteligencias múltiples Espacial y las variables emociones positivas en Física y Química $r=0,365(p=0,001)$, emociones positivas en Matemáticas $r=0,350(P=0,002)$ y emociones positivas en Ciencias Naturales $r=0,333(p=0,003)$.

Además, existe correlación significativa entre las inteligencias múltiples Corporal y las variables emociones positivas en Ciencias Naturales $r=0,363(p=0,001)$.

Observamos que también existen correlaciones significativas entre las inteligencias múltiples Naturaleza y las variables, emociones positivas en Matemáticas $r=0,260 \quad(P=0,022)$ y emociones positivas en Ciencias Naturales $r=0,477(p=0,000)$.

Y por último, vemos una correlación significativa entre las inteligencias múltiples Intrapersonal y la variable emociones positivas en Ciencias Naturales $r=0,229(p=0,046)$.

No obstante, no se producen correlaciones significativas entre las inteligencias múltiples 


\section{LAS EMOCIONES Y SU RELACIÓN CON LAS INTELIGENCIAS MÚLTIPLES EN LAS ASIGNATURAS DE CIENCIAS Y MATEMÁTICAS EN SECUNDARIA}

(Lingüística, Musical, Interpersonal e Intrapersonal) y las variables emociones positivas (en Física y Química, Matemáticas y Ciencias Naturales).

Tras comprobar los resultados obtenidos, podemos ver que la hipótesis planteada se cumple parcialmente, ya que no se cumple en todos los casos.

\section{CONCLUSIONES}

En este estudio hemos podido comprobar que la asignatura que más le gusta al alumnado en el curso de tercero de la ESO, de las tres que le hemos expuesto, es la de Ciencias Naturales.

En la asignatura de Física y Química obtienen una mayor puntuación en las emociones negativas como preocupación, nerviosismo y vergüenza. Entre las emociones positivas destaca es el entusiasmo.

El alumnado presenta más emociones negativas que positivas con respecto a la asignatura de Matemáticas. Las emociones negativas más destacadas son la preocupación y el nerviosismo; algunos alumnos manifiestan emociones positivas como confianza y tranquilidad.

Las puntuaciones más altas en emociones positivas la obtienen los alumnos en la asignatura de ciencias naturales destacando la alegría y la confianza. En las emociones negativas tan solo manifiestan un poco de preocupación y nerviosismo.

Con respeto a las inteligencias múltiples hemos obtenido variedad de resultados, y hemos comprobado que todos los alumnos destacan en algún tipo de inteligencia. En los campos que más destacan es en la inteligencia interpersonal, seguida de la corporal, lingüística e intrapersonal.

Con respecto a la hipótesis planteada en este estudio, sobre diferencias significativas entre las emociones que experimentan los alumnos en las distintas asignaturas y el aumento de un mayor porcentaje de algunas de las inteligencias múltiples, hemos observado que existe una correlación positiva entre las emociones positivas en Ciencias Naturales y una mayor puntuación en inteligencia Interpersonal y Naturalista.

Existen también correlaciones significativas entre las inteligencias múltiples Lógico Matemática y Espacial y las emociones positivas en Física y Química, en Matemáticas y en Ciencias Naturales.

\section{REFERENCIAS BIBLIOGRÁFICAS}

Bisquerra, R. (2000). Educación emocional y bienestar. Barcelona: Praxis

Bisquerra, R. (2003). Educación emocional y competencias básicas para la vida. Revista de Investigación Educativa, 21 (1), 7-43.

Borrachero, A.B., Brígido, M., Gómez R, Bermejo M.L. (2012). Relación entre autoconcepto y autoeficacia en los futuros profesores de Secundaria. International Journal of Developmental and Educational Psychology. INFAD 2 (1), 219-226

Borrachero, A.B. (2015). Las emociones en la enseñanza y el aprendizaje de las ciencias en educación secundaria. Tesis doctoral. Facultad de Educación. Badajoz: Universidad de Extremadura

Dávila, M. A., Borrachero, A. B., Brígido, M. y Costillo, E. (2014) Las emociones y sus causas en el aprendizaje de la Física y la Química. International Journal of Development and Educational Psychology. INFAD Revista de Psicología, 4(1), 287-295.

Damasio A. (2006). El error de Descartes, Madrid, Crítica,

Ekman, P. (1992). Facial expression and emotion. American Psychologist, 48 (4), 384392.

Fernández-Berrocal, P. y Ramos,N. (2002). Corazón y razón. En Fernández Berrocal, P. y Ramos, N. (Eds.). Corazones inteligentes. (17-34).Barcelona: Kairós.

Gardner, H. (1983). Frames of mind: The theory of Multiple Intelligences. New York: Basic Books.

Gardner, H. (1995). Inteligencias múltiples. La teoría en la práctica. Barcelona: Paidós.

Gardner, H. (2005) Inteligencias múltiples. Revista de Psicología y Educación, 1(1), 17-16 
Goleman, D. (1995). Inteligencia Emocional. Barcelona: Kairos.

Greenspan, S (1998). El crecimiento de la mente. Barcelona: Paidós.

Lucas Millán. C. (2013). Las emociones en ciencias y matemáticas y las inteligencias múltiples en Ios alumnos de Secundaria. Trabajo de master. Badajoz: Universidad de Extremadura.

Mayer, J., Salovey, P., Caruso, D. y Cherkasskiy, L. (2011). Emotional intelligence. The Cambridge handbook of intelligence, 528-599. New York.

Salovey, P. y Mayer, J.L. (1990). Emotional Intelligence. Imagination. Cognition and Personality, 9 (3), 185-211

Sosa, M. (2008). Escala autoinformada de inteligencia emocional (EAIE). Tesis doctoral. Universidad de Madrid. 
